Ann. Zootech., I980, 29 (4), 409-420.

\title{
Influence de la nature du régime alimentaire distribué aux poules sur la composition des stérols du jaune d'œuf
}

\author{
J. C. LHUGUENOT, B. COLAS, B. SAUVEUR $\left({ }^{*}\right)$ et D. BERTHEAU
}

École Nationale Supérieure de Biologie Appliquée à la Nutrition et à l'Alimentation (ENS. BANA) Campus Universitaire, 2I Ioo Dijon (France)

(*) Station de Recherches Avicoles, I.N.R.A., Tours, Nouzilly, $373^{80}$ Monnaie (France)

\section{Résumé}

Deux expériences ont été conduites dans le but d'étudier l'influence de l'incorporation de Io $\mathrm{p}$. Ioo de tourteau de colza ou de $6 \mathrm{p}$. I oo de protéines de luzerne dans la ration de poules pondeuses WARRFin ISA et STARCRoSs ShAVER sur la composition de la fraction stérolique des jaunes d'œufs.

L'analyse des stérols des régimes et des jaunes d'œufs a été effectuée par CCM — CGL précédée d'une extraction par solvants.

Les résultats montrent que :

- pour les niveaux d'incorporation pratiqués, la composition en stétols du tourteau de colza et des protéines de luzerne influence peu celle des régimes;

- il existe des variations dans l'œuf pour le cholestérol (expérimentation colza) et le 3 -sitostérol (expérimentation luzerne);

- le principal facteur de variation est l'origine génétique de la poule pondeuse : les œufs de WARREN sont plus riches en stérols que ceux de SHAVER (sauf en cholestérol) et sont également plus sujets à variations.

\section{Introduction}

lepuis une dizaine d'années, de nombreux travaux ont été consacrés à la recherche de nouvelles sources de protéines pouvant se substituer partiellement ou en totalité au tourteau de soja dans l'alimentation des poules pondeuses.

Dans une récente revue bibliographique, SAUveur (I980) présente les effets de différentes protéines : protéines végétales (légumineuses, protéines d'herbes, tourteau de colza), sous-produits industriels (solubles de distillerie, drêches de brasserie), farines animales et protéines d’organismes unicellulaires, sur les prin- 
cipaux paramètres qualitatifs de l'œuf. De telles substitutions peuvent entraîner des variations au niveau des oligo-constituants de l'œuf (NABER, I979).

Le présent travail a été consacré à la répercussion de 1'incorporation de tourteau de colza ou de protéines de luzerne dans le régime sur la composition de la fraction stérolique des lipides du jaune d'œuf. Nous nous sommes attachés à définir au mieux la fraction stérolique de ces deux matières premières ainsi que celle des rations expérimentales par rapport à la ration "soja témoin ». Nous avons ensuite analysé la fraction stérolique des oufs pondus lors de ces expérimentations dans le but de répondre à deux interrogations :

- Quelle est l'influence du régime alimentaire de la pondeuse sur le taux. de cholestérol du jaune? ClaRENBurG et al. (I97I) ont en effet montré qu'il existe une relation entre la teneur en $\beta$-sitostérol de la ration et le taux de cholestérol du jaune. Un dosage précis du cholestérol était donc rendu nécessaire et celui-ci devait tenir compte des interférences éventuelles avec d'autres stérols. Pour cette raison, la méthode classique de LIEBERMANN-BURCHARD a été rejetée au profit de la méthode par chromatographie gaz-liquide après dérivatisation des stérols.

- Une modification du régime peut-elle entraîner l'apparition ou la disparition d'un ou de plusieurs stérols dans le jaune? La démarche adoptée a alors consisté à suivre le profil chromatographique de la fraction stérolique et appliquer des méthodes fines d'identification, en l'occurrence le couplage chromatographie gaz-liquide - spectrométrie de masse, dans le cas de distorsions au niveau d'un ou de plusieurs stérols. Tu et al. donnent, en 1970 une identification très com:plète des stérols du jaune; on y trouve, en dehors d'une grande quantité de cholestérol, des teneurs importantes en desmostérol, cholestanol, ergostérol, $\beta$-sitostérol, $\Delta^{7}$-cholesténol, lanostérol, $\Delta^{7}$-méthosténol, 7-24-cholestadiène-4-4-diméthyl 3 $\beta$-ol,

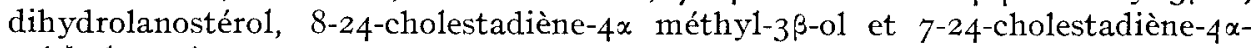
méthyl-3ß-ol.

\title{
Matériel et méthodes
}

\author{
Animaux, conditions d'élevage et collecte des aufs
} Nouzilly).

Ces étapes ont été conduites à la Station de Recherches Avicoles (I.N.R.A.,

Pour l'expérimentation "colza ", les animaux étaient des poules de souches WARREN ISA (productrices d'œufs colorés) et STARCROSS SHAVER (productrices d'œufs blancs), âgées de 60 semaines en début d'expérience, et recevaient ad libitum soit un régime témoin à base de tourteau de soja, soit un régime expérimental contenant io p. roo de tourteau de colza.

Pour l'expérimentation "protéines de luzerne ", les animaux étaient des poules de souches WARREN ISA, âgées de 60 semaines en début d'expérience, et recevaient ad libitum soit un régime témoin à base de tourteau de soja, soit un régime expérimental contenant $6 \mathrm{p}$. Ioo de protéines de luzerne PXI.

La composition des régimes est donnée dans le tableau I.

Pour l'expérimentation colza, les œufs ont été prélevés au jour o de la distribution du régime contenant ro p. Ioo de tourteau de colza et après $2,3,4$ et 5 semaines d'adaptation. Pour l'expérimentation luzerne, les œufs ont été prélevés après 2,3 et 4 semaines d'adaptation au régime. 


\section{TABLEAU I}

Composition des régimes utilisés (p. Ioo) (B. Sauveur et J. C. BLum, Station de Recherches Avicoles, I.N.R.A., Nouzilly)

Ingredients of diets ( $p$. Ioo)

\begin{tabular}{|c|c|c|c|c|}
\hline & \multicolumn{2}{|c|}{$\begin{array}{l}\text { Expérimentation } \\
\text { Colza } \\
\text { (Rapeseed experiment) }\end{array}$} & \multicolumn{2}{|c|}{$\begin{array}{c}\text { Expérimentation } \\
\text { Prot. de luzerne } \\
(\text { Lucerne protein expériment })\end{array}$} \\
\hline & $\begin{array}{l}\text { Témoin } \\
\text { Control }\end{array}$ & $\begin{array}{l}\text { Colza } \\
\text { Io p. Ioo }\end{array}$ & Témoin & $\begin{array}{l}\text { Luzerne } \\
6 \text { p. 10o }\end{array}$ \\
\hline 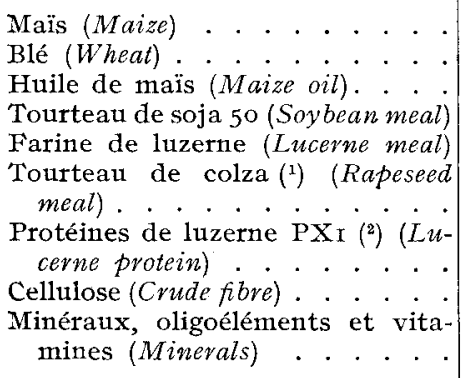 & $\begin{array}{r}39,8 \\
28,0 \\
- \\
\mathrm{I} 7,5 \\
4,0 \\
- \\
- \\
- \\
\mathrm{I} 0,7\end{array}$ & $\begin{array}{r}34,5 \\
26,0 \\
2,5 \\
12,0 \\
4,0 \\
10,0 \\
- \\
0,4 \\
10,6\end{array}$ & $\begin{array}{r}20,0 \\
48,0 \\
1,0 \\
17,0 \\
- \\
- \\
- \\
4,0 \\
10,0\end{array}$ & $\begin{array}{r}20,0 \\
48,0 \\
1,0 \\
11,0 \\
- \\
- \\
6,0 \\
4,0 \\
10,0\end{array}$ \\
\hline
\end{tabular}

(I) Colza o acide érucique.

(2) France Luzerne, II rue de Madrid, 75005 Paris.

\section{Analyse qualitative et quantitative des fractions stéroliques}

Ces analyses ont porté sur les régimes "témoins ", "colza " et " protéines de luzerne PXI ", ainsi que sur leurs composants : blé, maïs, tourteau de soja, farine de luzerne, tourteau de colza et protéines de luzerne PXI.

Les fractions stéroliques des farines et des jaunes d'œufs sont obtenues grâce à une technique d'extraction par solvants (chloroforme/méthanol $2 / \mathrm{I}$ pour les farines, méthylal /méthanol 4 /I pour les jaunes) suivie d'une saponification (OHAVERON, I966). L'insaponifiable est ensuite fractionné par chromatographie sur couche mince (Prosi et al., I 974) et les stérols sont analysés par chromatographie gazeuse. Cette association CCM - CGL a été proposée par THorpe et al. (rg69) pour l'analyse du cholestérol dans les huiles et les graisses.

En I970, THORPE compare différentes techniques de séparation des stérols par chromatographie gazeuse. On y relève les travaux de VAN DEN HEUVÉ et al. (r960), VAN den Heuvel, \& Horning (ig62), Bloomfield (ig62), La Croix (ig69) et Grunwald (I969) dans lesquels l'analyse en CGL se fait sur des stérols libres. Par contre, dès I965, EtTINGGE et al. (I965) proposent une dérivatisation préalable de ces composés sous forme d'acétate. Enfin, TatTrie (I972) décrit une métỉode 
d'extraction et de dosage du cholestérol dans le jaune d'œuf sous forme d'éther de triméthylsilyle.

Dans la présente étude, l'analyse des stérols par chromatographie gazeuse a été effectuée à l'aide de leurs dérivés triméthylsilylés (LuUKKAINEN et al., I96I; VAN DEN HEUVEI, \& CoURT, I968).

L'appareil est un chromatographe PACKARD 409 équipé de deux détecteurs à ionisation de flamme. Les conditions expérimentales étaient les suivantes :

- colonnes en verre, de diamètre $3 \mathrm{~mm}$ et de longueur $4 \mathrm{~m}$, silanisées, remplies d'OV-I ou d'OV-I7 à I p. roo sur Chromosorb W AW-DMCS roo-I 20 mesh (Spiral, rue des Roses, 2 I0oo Dijon);

- température des colonnes : programmation de $0,8{ }^{\circ} \mathrm{C} / \mathrm{mn}$ à partir de $230^{\circ} \mathrm{C}$;

- température des injecteurs : $240^{\circ} \mathrm{C}$;

- température des détecteurs : $290^{\circ} \mathrm{C}$;

- gaz vecteur : azote dont la pression d'entrée est de $1,75 \mathrm{~kg} / \mathrm{cm}^{2}$ à $230{ }^{\circ} \mathrm{C}$;

- débit d'hydrogène : $25 \mathrm{ml} / \mathrm{mn}$;

- intégrateur Hewlett-Packard $3380 \mathrm{~A}$.

L'analyse quantitative a été réalisée par la méthode de l'étalon interne (épicoprostanol) qui a été ajouté à l'échantillon en début d'extraction. Les rendements d'extraction et de dérivatisation sont considérés comme identiques pour les différents stérols étudiés et l'épicoprostanol (Prost, Maume et Padieu, I974).

\section{Résultats et discussion}

\section{Fraction stérolique des régimes et de leurs composants}

Le tableat 2 donne la composition en stérols des matières premières utilisées et des régimes. Les résultats sont exprimés en $u g / g$ et correspondent à la phase OV-I7 qui a donné la meilleure séparation. Un exemple de profil chromatographique est donné figure I. Certains pics séparés ont pu être identifiés grâce à leur indice de méthylène comparé à celui des stérols de référence. Dans tous les échantillons analysés ont été trouvés les stérols suivants : cholestérol, campestérol, stigmastérol et $\beta$-sitostérol. I,es autres pics séparés (appelés $A, B, C, D$ et $E$ ) ont été analysés en spectrométrie de masse. Le pic $A$, présent uniquement dans le tourteau de colza et le régime colza correspond au brassicastérol.

Les fractions stéroliques du blé, du maïs et du tourteau de soja sont qualitativement identiques, la richesse en stérols allant croissant dans l'ordre : tourteau de soja, maïs, blé. On peut noter la présence en quantité importante du brassicastérol dans le tourteau de colza, ce stérol se retrouvant en quantité moindre dans le régime correspondant $\mathrm{d} u$ fait que la teneur en colza n'y est que de ro p. Ioo. Les composants farine et protéines de luzerne présentent un caractère important : une diminution très nette de la teneur en $\beta$-sitostérol. Les composés $\mathrm{B}, \mathrm{C}$ et $\mathrm{F}$, représentent alors la majeure partie de la fraction stérolique. Il semble donc y avoir dans ce cas un équilibre différent au niveau des phytostérols. Ce caractère important s'estompe en partie dans le régime "protéines de luzerne PXI " du fait de 1'apport des autres constituants.

Plus généralement, les variations observées dans les matières premières sont peu visibles au niveau des régimes, qualitativement et quantitativement. Le 
PROTEINE ALIMENTAIRE ET STÉROLS DE L'GEFF

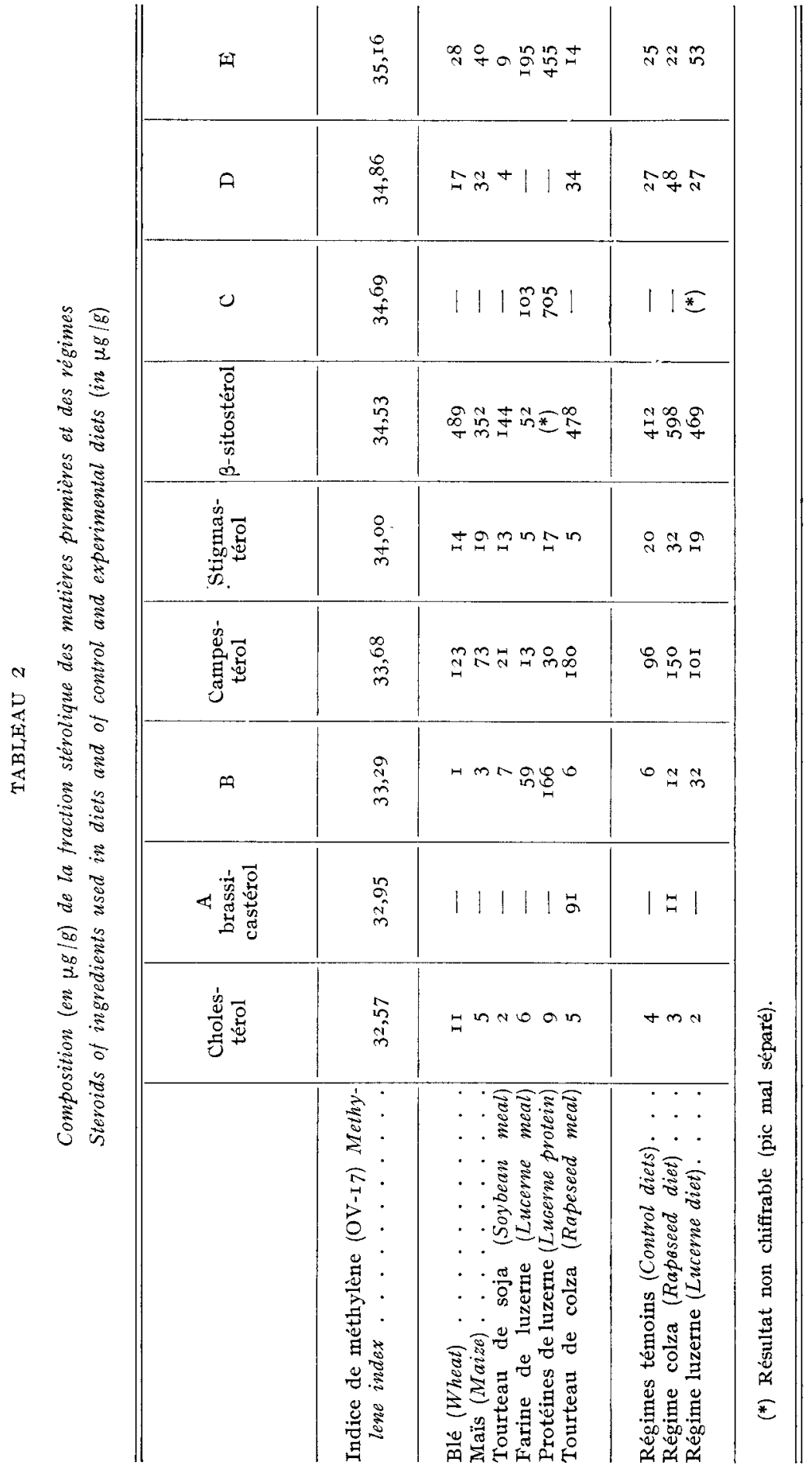




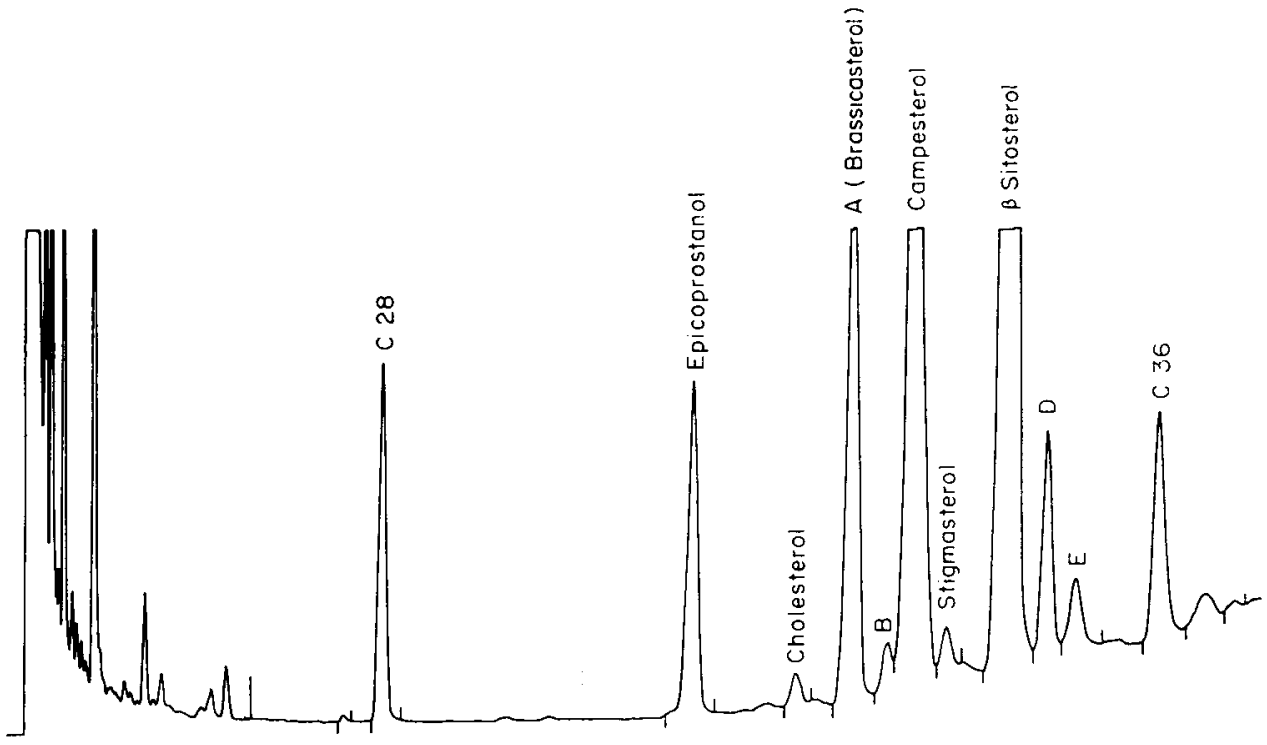

FIG. I. - Profil en chromatographie gaz-liquide de la fraction stérolique du tourteau de colza (phase OV 17 - atténuation $3,2.10^{9} \mathrm{~A}$ )

Chromatographic profile of the sterol fraction extracted from rapesed meal

fait que le tourteau de colza et les protéines de luzerne n'entrent respectivement que pour Io p. Ioo et $6 \mathrm{p}$. Ioo dans les régimes explique un tel résultat. Ces taux d'incorporation peuvent sembler faibles; ils entraînent pourtant des altérations de 1a qualité des oufs : apparition de flaveurs désagréables avec le tourteau de colza, discoloration du jaune avec les protéines de luzerne.

\section{Fraction stérolique des ceufs}

Un exemple de profil chromatographique est donné figure 2 . Cette étude nous a amené à séparer II pics (repérés $a$ à $k$ ) dont 2 correspondent au cholestérol et au $\beta$-sitostérol.

Les résultats des compositions en stérols des œufs sont donnés dans les tableaux 3 (régimes témoins), 4 (expérimentation colza) et 5 (expérimentation luzerne). Trois stérols ( $d, h$ et $k$ ) n'ont pu être quantifiés car mal séparés ou en trop faible quantité. I es teneurs sont exprimées en $\mu \mathrm{g} / \mathrm{g}$ de poids frais.

Les teneurs en cholestérol sont comparables à celles trouvées dans la littérature (Martin, TATTRIE et CoOK, I963; RANGachar, SetTy et Hedge, i97o; TURK et BARNETT, I97I; FEELEY, CRINER et WATT, I972; TATTRIE, I972; CUNNINGHAM et al., I974; Nix et al., I974; WASHBURN et Nix, I974).

Pour les régimes témoins (tabl. 3) la teneur en cholestérol des œufs de WARREN ne diffère pas de celle des œufs de SHAvEr. En dépit d'écarts-types élevés, ce qui semble traduire de fortes variations individuelles au niveau des animaux d'un même lot et au cours du temps, des différences significatives sont observées 


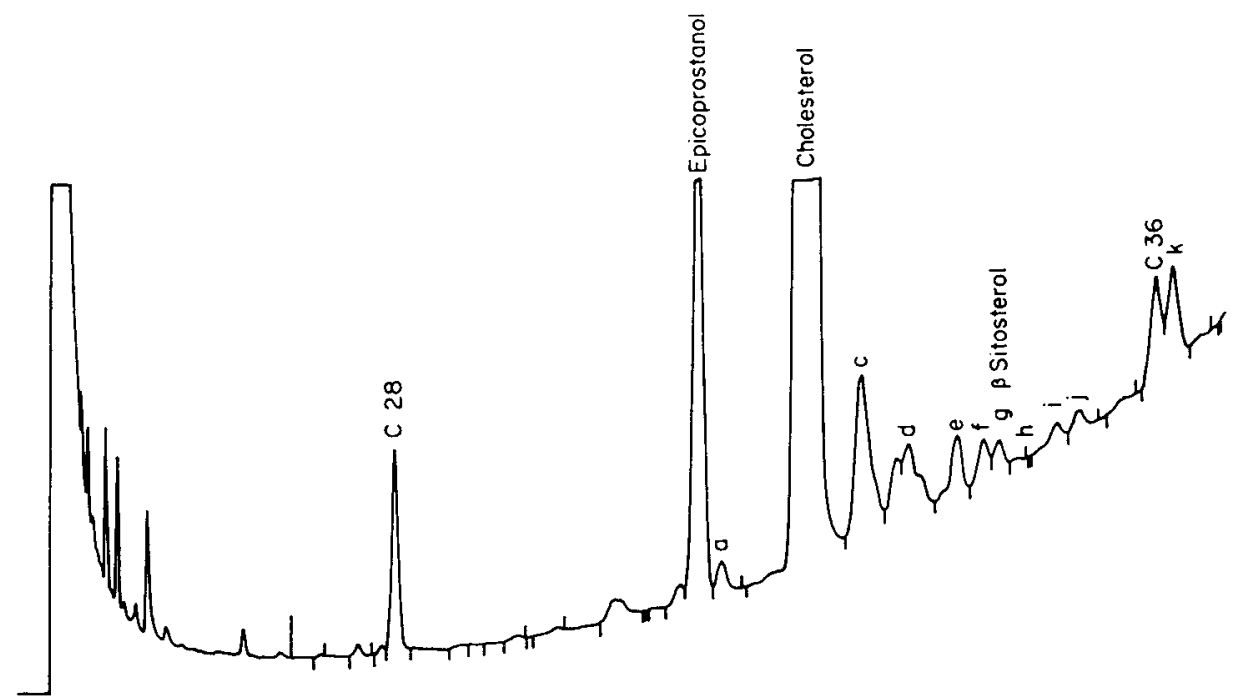

FIG. 2. - Profil chromatographique de la fraction stérolique du jaune d'ceuf obtenu avec le régime colza (souche Warren - 34 $4^{\mathrm{e}}$ ) (phase OV I7 - atténuation $8.10^{-10} A$ ) Chromatographic profile of the sterol fraction (Warren egg yolk obtained after 34 days of rapeseed meal diet).

pour les stérols $a, c, e$ et $f$. Ces différences sont toujours dans le sens d'une plus faible teneur dans les oufs de poules SHaver.

Dans l'expérimentation colza (tabl. 4), les profils chromatographiques obtenus montrent que le brassicastérol présent dans le régime n'est pas retrouvé dans les œufs. Par ailleurs, les différences observées en fonction de la durée d'adaptation au régime varient selon le type génétique de la poule :

- pour la souche WARREN, le taux de cholestérol diminue à partir de quatre semaines d'adaptation et les teneurs en stérols $a, i$ et $j$ sont élevées après 4 semaines mais ne diffèrent pas de celles des témoins après 5 semaines;

- pour la souche SHAVER, aucune évolution du taux de cholestérol n'est observée et des différences sont mises en évidence pour les stérols $e$ et $f$ sans qu'il soit possible de conclure à une évolution. Plus généralement, les variations observées pour cette souche sont moins nombreuses que pour la souche WARREN et les plus faibles teneurs en stérols $a, c$, et $t$, mises en évidence pour les régimes témoins (tabl. 3) sont conservées.

Dans l'expérimentation luzerne (tab1. 5), la seule variation observée concerne une augmentation de la teneur en $\beta$-sitostérol après quatre semaines d'adaptation. Cette augmentation ne peut être expliquée par la composition du régime luzerne qui, pour ce stérol, ne diffère pas de celle du régime témoin (tabl. 2 ).

L'ensemble de ces résultats montre que, pour les niveaux d'incorporation pratiqués, la composition en stérols du tourteau de colza et des protéines de luzerne influence peu celle des régimes. Il en résulte de faibles variations qualitatives et quantitatives des fractions stéroliques des jaunes produits : seules les teneurs en $\beta$-sitostérol (dans le cas du régime luzerne) et en cholestérol (dans le cas du régime colza) présentent une évolution; encore faut-il préciser que la diminution de teneur 


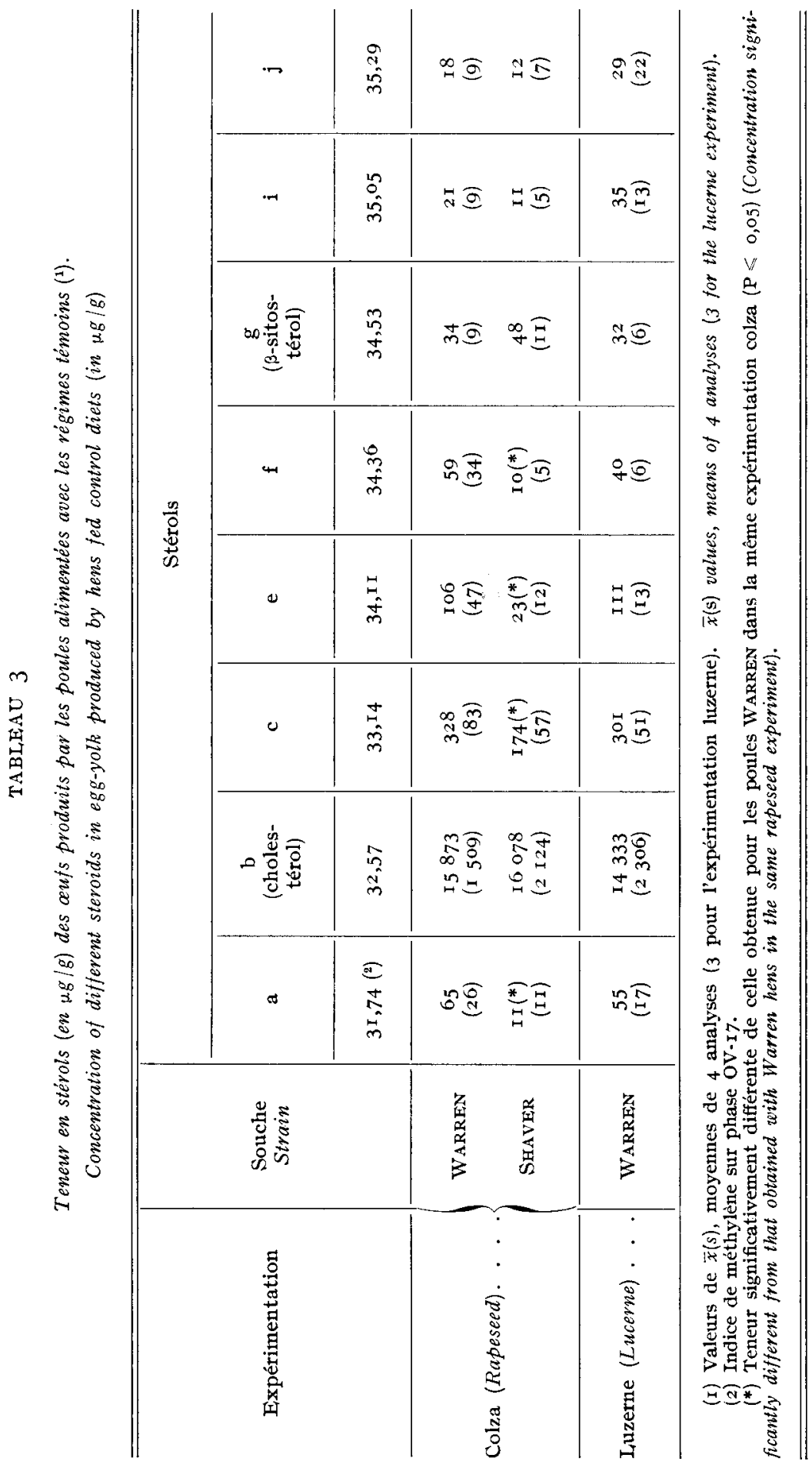




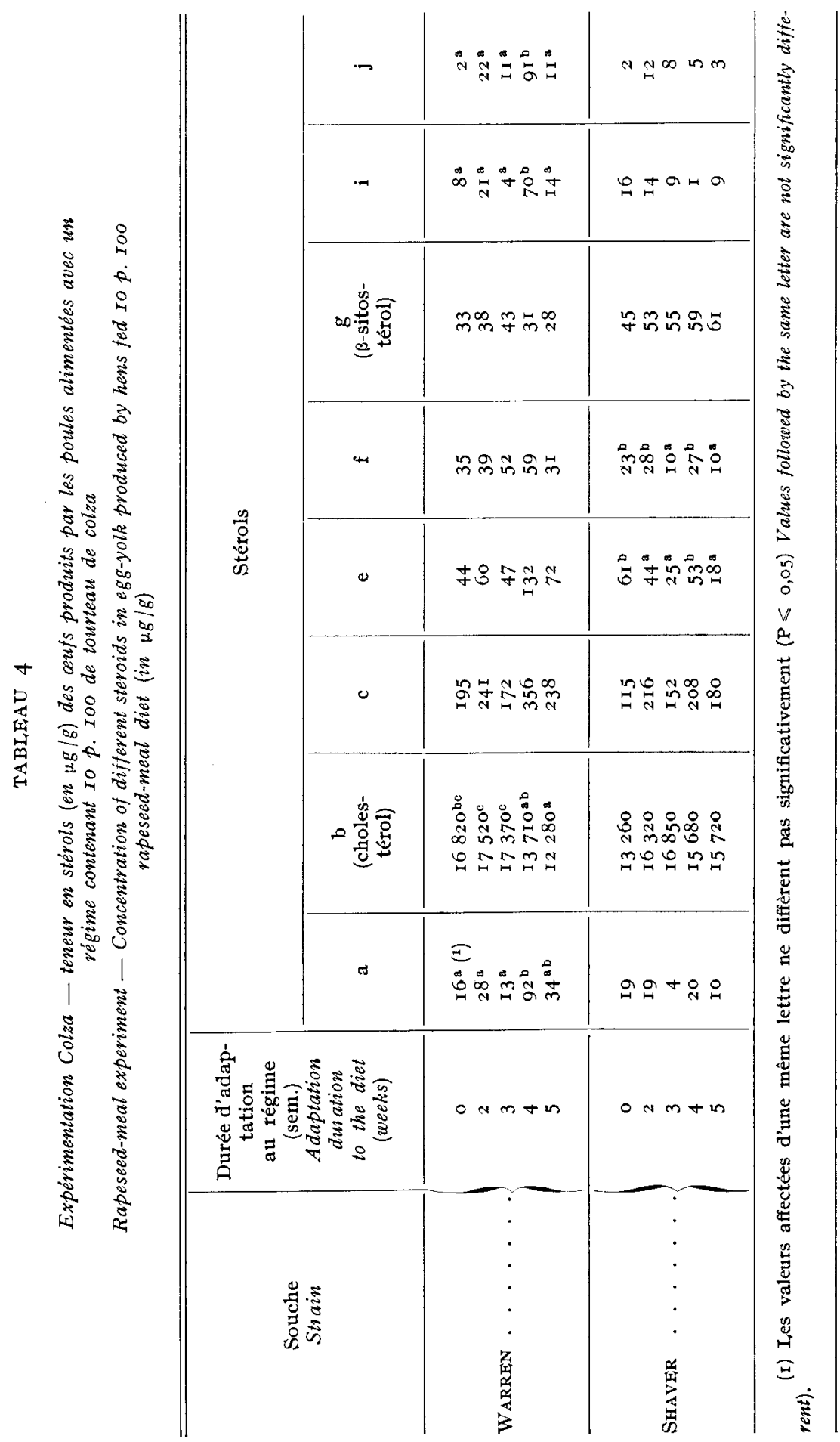




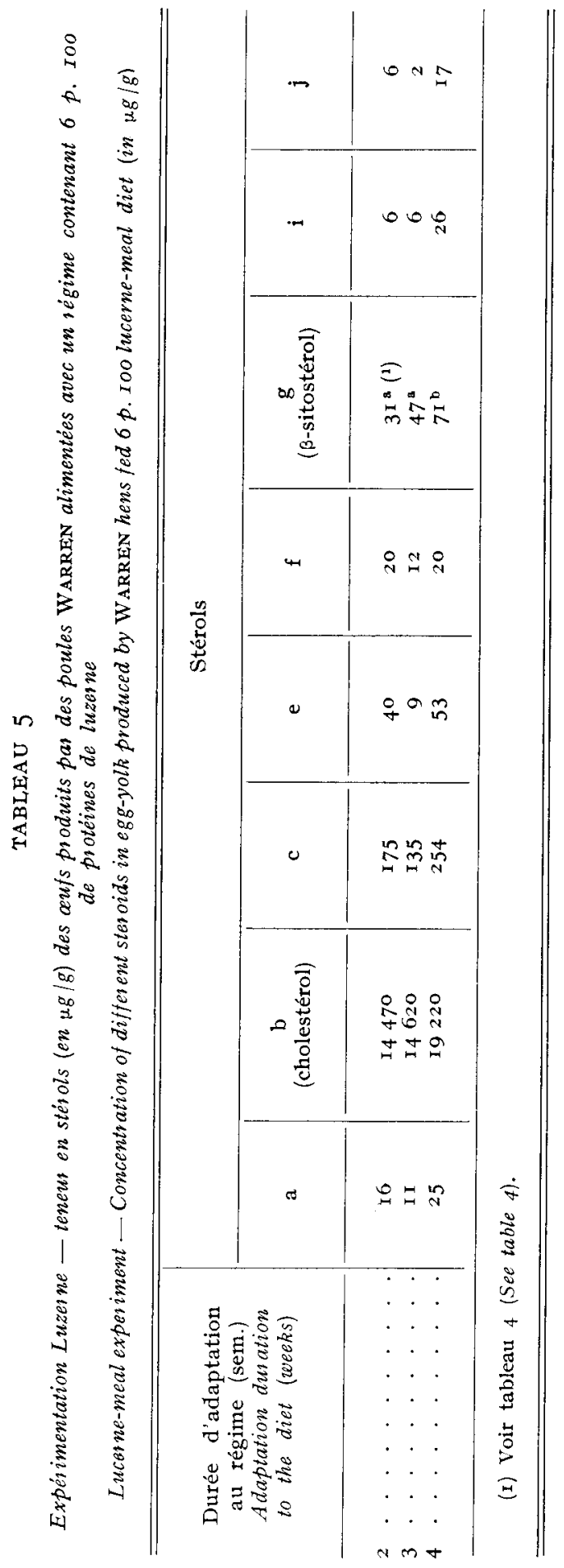


en cholestérol ne concerne que les œufs de poules WARREN. Cette souche apparaît d'ailleurs globalement plus dépendante de la composition des régimes et les œufs produits sont plus riches en stérols autres que le cholestérol que ceux produits par la souche SHAVER.

Accepté pour publication en décembre 1980.

\section{Summary \\ Effect of the dietary protein fraction on the profile of some egg yolk steroids}

Experiments were made to study the influence of incorporating rapeseed meal (Io p. Ioo) or lucerne proteins (6 p. Ioo) to laying hen diets on the profile of the sterol fraction of egg yolk lipids. WARREN ISA and Starcross SHAver hens laying brown or white-shelled eggs, respectively were used.

The sterol fraction of control (containing I 7 p. Ioo soybean meal) and experimental diets were analysed by thin-layer and gas liquid chromatography after liquid partition.

The results indicate:

- a slight difference in the phytosterol profile of experimental and control diets (Tab1. 2);

- a variation in the egg yolk level of cholesterol (rapeseed 'experiment - tabl. 4) and $\beta$-sitosterol (lucerne experiment - tabl. 5);

- that the most important variation factor inducing a sterol profil difference is a genetic factor (WARREN eggs have a higher sterol content than SHAVER eggs, except for cholesterol).

\section{Remerciements}

Les travaux rapportés ont été réalisés avec l'aide financière de la D.G.R.S.T., Action Concertée Technologie Alimentaire et Agricole, décision $\mathrm{n}^{0}$ 78.7.0455.

\section{Références bibliographiques}

BLOOMFIELD D. K., I 962 . Quantitative analysis of complex mixtures of steroids and bile acids. Anal. Chem., 34, 737-745.

Chaveron H., I966. Détermination de la composition stérolique du beurre de cacao. Chocolaterie, Confiserie de France, 218, I3-22.

Clarenburg R., Kim Chung I. A., Wakefield L. M., I97 I. Reducing the egg cholesterol level by including emulsified sitosterol in standard chick diet. $J$. Nutv., 101, 289-297.

Cunningham D. L., KReUger W. F., Fanguy R. C., Bradley J. W., i974. Preliminary results of bidirectional selection for yolk cholesterol level in laying hens. Poult. Sci., 53, 384-392.

EtTinger C. I., Malonoski A. J., Kirschenbaum H., i965. Detection and estimation of animal fats in vegetable oils by gas chromatography. J. Assoc. Off. Anal. Chem., 48, I I86-I 192.

FeEley R., Criner P. E., Wa'tr B. K., 1972. Cholesterol content of foods. J. Am. Diet. Assoc., 61, I34-I39.

GRUNWALD C., r969. Analysis of free phytosterols by gas chromatography using liquid phase OV-Ior. J. Chromatog., 44, I 73-1 78 .

LA Crorx D., 1969. Extraction of plant sterols from adulterated butter oil using a digitoninimpregnated celite colum. $J$. Assoc. Off. Anal. Chem., 52, 600-608.

I,UUkKainen T., Van Den Heuver, W. J. A., HaAhti E. O. A., Horning E. C., I96i. Gas chromatographic behaviour of trimethylsilyl esters of steroids. Biochem. Biophys. Acta, 52, $599-6$ r 4 . 
Martin W. G., TATrTRE N. H., CoOK W. H., I963. Lipid extraction and distribution studies of egg yolk lipoproteins. Can. J. Biochem. Physiol., 41, I57-r7o.

NABER E. C., 1979. The effect of nutrition on the composition of eggs. Poult. Sci., 58, 518-528.

Nix D. F., Thornton E. J., Washburn K. W., Marks H. L., I974. The influence of moulting on yolk cholesterol level. Poult. Sci., 53, 412-416.

Prost M., Maume B. F., Padieu P., I974. Identification de phytostérols, d'oxo- et d'hydroxycholesterols par chromatographie gaz-liquide couplée à la spectrométrie de masse. Biochem. Biophys. Acta, 360, 230-24I.

Rangachar T. R. S., SeTtTy S. V. S., HedGe R., 1970. Cholesterol content in eggs of chicken and duck. Mysore J. Agric. Sci., 4, I46-I52.

Sauveur B., I980. Matières premières protéiques et qualités de l'œuf. In "Matières premières et Alimentation des volailles ", St. de Rech. Avicoles Edit., Nouzilly, I 73-I9I.

TATTRIE N. H., 1972. Isolation and identification of egg yolk cholesterol esters. Can. J. Biochem., 50, I 4 I 4-I 423 .

Thorpe C. W., Pohland L., Firestone D., ig69. Thin-layer and gas-liquid chromatography of cholesterol in fats and oils. I. Development of method. J. Assoc. Off. Anal. Chem., 52, $774-780$.

THORPE C. W., I970. Comparison of separation procedures for identification of oils by gas chromatography. J. Assoc. Off. Anal. Chem., 53, 623-63I.

TU C., Powrie W. D., Fennema O., i 97o. Steroids in egg yolk. J. Food. Sci., 35, 6oi-606. TURK D. E., BARNETT B. D., I97I. Cholesterol content of market eggs. Poult. Sci., 50, I303-I 307.

Van Den Heuvel, W. J. A., Sweeley C. C., Horning E. C., ig6o. Separation of steroids by gas chromatography. J. Am Chem. Soc., 82, 348I-3486.

VAN DEN HEUVEL W. J. A., HORNING E. C., I962. A study of retention time relationships in gas chromatography in terms of the structure of steroids. Biochem. Biophys. Acta, 64, 4I6-423.

VAN DEN HeUver, W. J. A., CoURT A. S., I968. Reference high-efficiency nonpolar packed columns for the gas-liquid chromatography of nanogram amounts of steroids. $J$. Chromatog., 38, 439-448.

Washburn K. W., Nix D. F., I974. Genetic basis of yolk cholesterol content. Poult. Sci., 53, I $\operatorname{log-I} 20$. 